$02,11,12$

\title{
Переход зарядовый порядок-сверхтекучесть в двумерной системе локальных бозонов и возникающие доменные структуры
}

\author{
() А.С. Москвин ${ }^{1}$, Ю.Д. Панов ${ }^{1}$, Ф.Н. Рыбаков ${ }^{1,2}$, А.Б. Борисов ${ }^{1,2}$ \\ ${ }^{1}$ Уральский федеральный университет им. Б.Н. Ельцина, \\ Екатеринбург, Россия \\ ${ }^{2}$ Институт физики металлов им. М.Н. Михеева УрО РАН, \\ Екатеринбург, Россия \\ E-mail: alexander.moskvin@urfu.ru
}

\begin{abstract}
Мы использовали высокопроизводительные параллельные вычисления на графических картах NVIDIA методом нелинейных сопряженных градиентов и методом Монте-Карло, чтобы непосредственно наблюдать формирование конфигурации основного состояния двумерной системы локальных бозонов с понижением температуры и ее эволюции с отклонением от половинного заполнения. Это позволило нам исследовать необычные особенности фазового перехода зарядовый порядок-сверхтекучесть, в частности, формирование нерегулярной доменной структуры, возникновение нитевидной сверхтекучей структуры, конденсирующейся в антифазных границах доменов зарядово-упорядоченной фазы, а также формирование и эволюцию различных топологических структур.
\end{abstract}

Работа выполнена при поддержке программы 211 правительства Российской Федерации, соглашение № 02.А03.21.0006 и проектов № 2277 и № 5719 Министерства образования и науки Российской Федерации.

DOI: $10.21883 /$ FTT.2017.11.45045.04k

\section{1. Введение}

Модель квантового решеточного бозе-газа и локальных бозонов имеет долгую историю и первоначально была использована для обычных сверхпроводников и квантовых кристаллов, таких как ${ }^{4} \mathrm{He,} \mathrm{где} \mathrm{сверхтеку-}$ честь сосуществует с кристаллическим порядком. Затем модель Бозе-Хаббарда была использована как модель перехода сверхпроводник-изолятор в системах с локальными бозонами, биполяронами или предварительно сформированными куперовскими парами, а также для описания анизотропных квантовых магнетиков, пленок сверхтекучего гелия и фазы сверхтекучего твердого тела. Двумерные модели Бозе-Хаббарда использовались для описания сверхпроводящих пленок и массивов джозефсоновских контактов. Самый недавний интерес к системе локальных бозонов связан с выдающимися результатами по бозе-эйнштейновским конденсатам атомных систем, полученных путем захвата нейтральных атомов в оптической решетке. Тем не менее, мы хотели бы напомнить и подчеркнуть тесную связь модели локальных бозонов с физикой ВТСП-купратов как в нормальном, так и в сверхпроводящем состоянии (см., например, [1]). С приходом эры спиновых флуктуаций и квантовых осцилляций интерес к этой модели заметно остыл, и она была незаслуженно забыта. Мы полагаем, что природа особых свойств купратов может быть связана с наблюдаемыми в них высокой диэлектрической проницаемостью и сильными электронно-решеточными поляризационными эффектами, а также с их неустойчивостью по отношению к конденсации экситонов с $d-d$ переносом заряда $[2,3,4]$ и эволюцией в электронно-дырочную (ЭД) систему. В отличии от электронно-дырочной жидкости в полупроводниках, которая представляет собой двухкомпонентную ферми-жидкость, ЭД-система в купратах представляет собой систему локализованных электронных $\left[\mathrm{CuO}_{4}^{7-}\right]$ и дырочных $\left[\mathrm{CuO}_{4}^{5-}\right]$ центров, или, номинально, $\mathrm{Cu}^{1+}$ и $\mathrm{Cu}^{3+}$ ионов с двухчастичным бозонподобным транспортом. Такая ЭД-система эквивалентна системе локальных бозонов $[2,3,4]$. Реальная фазовая диаграмма $T-x$ для ВТСП-купратов отражает результат борьбы в основном состоянии между двумя простыми фазами: бозонной $\mathrm{Cu}^{1+}-\mathrm{Cu}^{3+}$ ЭД-системой и фермионной системой $\mathrm{Cu}^{2+}-\mathrm{Cu}^{3+}$ или $\mathrm{Cu}^{2+}-\mathrm{Cu}^{1+}$, в частности, конкуренции между двух- и одночастичным транспортом с сопротивлением $\propto T$ и $\propto T^{2}$ соответственно. Для описания более сложной бозонфермионной системы $\mathrm{Cu}^{1+}-\mathrm{Cu}^{2+}-\mathrm{Cu}^{3+}$ можно использовать псевдоспиновый $(S=1)$ формализм (модель зарядового триплета) $[2,3,4,5,6]$ или более традиционные бозонфермионные сценарии (см., например, $[7,8]$ ).

По аналогии с обычными спиновыми системами, псевдоспиновый формализм $(S=1)$ позволяет предсказать различные типы диагонального и недиагонального дальнего порядока и псевдоспиновых возбуждений, в том числе, соизмеримый и несоизмеримый зарядовые порядки (волны псевдоспиновой плотности), фазы сверхтекучей жидкости и сверхтекучего твердого тела, различные топологические возбуждения, характерные для двумерных систем, в частности, новый тип топологических дефектов для родительских купратов [5].

Чисто бозонный бесспиновый сценарий для допированных купратов является очевидным упрощением, однако он позволяет описать многие характерные черты зарядовой степени свободы в нормальном и сверхпроводящем состояниях, в том числе, необычную псевдощеле- 
вую фазу $[2,3,4,6]$, которая может быть связана с типичной для системы локальных бозонов фазой зарядового порядка. Простейший пример перехода от зарядового упорядочения к сверхтекучей жидкости реализуется в двумерной модели заряженных локальных бозонов.

В настоящей работе мы использовали высоко производительные параллельные вычисления на графических картах NVIDIA, чтобы непосредственно наблюдать формирование конфигурации основного состояния двумерной системы локальных бозонов с понижением температуры и ее трансформацию с ростом отклонения от половинного заполнения, при котором корреляционноиндуцированная изолирующая фаза с локализованными частицами трансформируется в сверхтекучую фазу.

\section{2. Модель локальных бозонов}

Гамильтониан локальных (hard-core) бозонов имеет стандартный вид $[9,10,11,12]$

$$
H_{h c}=-\sum_{\langle i j\rangle} t_{i j} \hat{P}\left(\hat{b}_{i}^{\dagger} \hat{b}_{j}+\hat{b}_{j}^{\dagger} \hat{b}_{i}\right) \hat{P}+\sum_{\langle i j\rangle} V_{i j} n_{i} n_{j}-\mu \sum_{i} n_{i},
$$

где проекционный оператор $\hat{P}$,запрещает“ двукратное заселение любого узла решетки, $\hat{b}^{\dagger}(\hat{b})$ - операторы Паули рождения (уничтожения) с коммутационными соотношениями Бозе для разных узлов $\left[\hat{b}_{i}, \hat{b}_{j}^{\dagger}\right]=0$, если $i \neq j,\left[\hat{b}_{i}, \hat{b}_{i}^{\dagger}\right]=1-2 n_{i}, n_{i}=\hat{b}_{i}^{\dagger} \hat{b}_{i} ; N-$ полное число узлов, $\mu-$ химический потенциал, определяемый из условия фиксированного числа бозонов $\sum_{i=1}^{N}\left\langle n_{i}\right\rangle, t_{i j}-$ эффективный интеграл переноса, $V_{i j}$ - межцентровое взаимодействие бозонов. Ниже мы рассматриваем перенос и бозон-бозонное отталкивание только между ближайшими соседями, $V_{i j}=V_{n n}=V>0, t_{i j}=t_{n n}=t>0$. Стоит отметить, что вблизи половинного заполнения, когда концентрация $n=\frac{N}{1} \sum_{i=1}^{N}\left\langle n_{i}\right\rangle \approx 1 / 2$, можно ввести перенормировку $n_{i} \rightarrow n_{i}-1 / 2$, или нейтрализующий фон, что сразу обеспечивает симметрию частица-дырка. Система локальных бозонов с отталкиванием на соседних узлах эквивалентна квантовому магнетику с постоянной намагниченностью - системе спинов $s=1 / 2$ во внешнем поле, направленном вдоль оси $z$ [9]. При учете нейтрализующего фона система описывается эффективным псевдоспиновым гамильтонианом

$$
H_{h c}=\sum_{\langle i j\rangle} J_{i j}^{x y}\left(\hat{s}_{i}^{+} \hat{s}_{j}^{-}+\hat{s}_{j}^{+} \hat{s}_{i}^{-}\right)+\sum_{\langle i j\rangle} J_{i j}^{z} \hat{s}_{i}^{z} \hat{s}_{j}^{z}-\mu \sum_{i} \hat{s}_{i}^{z},
$$

где $J_{i j}^{x y}=2 t_{i j}, J_{i j}^{z}=V_{i j}, \hat{s}_{i}^{-}=\frac{1}{\sqrt{2}} \hat{b}_{i}, \hat{s}_{i}^{+}=-\frac{1}{\sqrt{2}} \hat{b}_{i}^{\dagger}, \hat{s}_{i}^{z}=$ $=-\frac{1}{2}+\hat{b}_{i}^{\dagger} \hat{b}_{i}, \hat{s}_{i}^{ \pm}=\mp \frac{1}{\sqrt{2}}\left(\hat{s}_{i}^{x} \pm i \hat{s}_{i}^{y}\right)$.

Локальный порядок в такой системе описывается тремя параметрами: $\left\langle\hat{s}^{z}\right\rangle=n-1 ;\left\langle\hat{s}^{ \pm}\right\rangle=\left|\left\langle\hat{s}^{ \pm}\right\rangle\right| e^{ \pm i \varphi}$, связанными с зарядовой и сверхтекучей степенями свободы соответственно.
При половинном заполнении реализуются две фазы: „диагональный“ зарядовый порядок (charge order, CO) или „недиагональный“ сверхтекучий (superfluid, SF) дальний порядок при $V>2 t$ или $V<2 t$ соответственно. Однако при отклонении от половинного заполнения многочисленные исследования указывают на возникновение фазы сверхтекучего твердого тела (supersolid, SS) или фазы $\mathrm{CO}+\mathrm{SF}$ с одновременно не равными нулю „диагональным“ и „недиагональным“ параметрами порядка (см. ссылки в $[10,11,12])$.

На рис. 1 представлена фазовая диаграмма системы локальных бозонов на квадратной решетке с интегралом переноса поближайшим соседям $t_{n n}=t$ для $V_{n n}=V=3 t \quad\left(J^{z}=(3 / 2) J^{x y}\right)$, построенная по результатам расчетов методом квантового Монте-Карло (QMC) из работы [12]. При половинном заполнении система переходит в СО-фазу при $T_{\mathrm{CO}} \approx 0.5 \mathrm{~V}=1.5 t \quad[12]$. Отклонение $\Delta n$ от половинного заполнения приво-

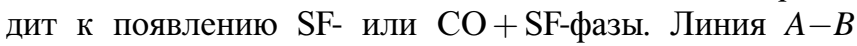
показывает температуру $T_{K T}(\Delta n)$ фазового перехода Березинского-Костерлица-Таулеса; линия $C-B-D-C$ соответствует фазовому переходу первого рода; линия $D-E$, которая может быть названа температурой псевдощели $T^{*}(\Delta n)$, соответствует фазовому переходу второго рода $\mathrm{CO}-\mathrm{NO}$ в неупорядоченную (non-ordered, NO) фазу. Важно отметить, что SS-фаза, существующая при отклонении $\Delta n$ от половинного заполнения как стабильная в приближении среднего поля [1], оказывается термодинамически неустойчивой по отношению к фазовому разделению на CO- и SF-фазы согласно результатам расчетов методом QMC [12].

Фазовая диаграмма локальных бозонов в приближении среднего поля хорошо известна (см., например, [1]). Прежде всего при отклонении $\Delta n$ от половинного заполнения при $T=0$ и $V>2 t$ появляется однородная SSфаза, которая при критической концентрации

$$
\Delta n_{c}=\frac{1}{2}\left(\frac{V-2 t}{V+2 t}\right)^{\frac{1}{2}}
$$

переходит в SF-фазу. При $V=3 t$ значение $\Delta n_{c}=$ $=n_{c r} \approx 0.11$, которое получается из расчетов методом QMC [12]. Температура перехода между фазами NO и $\mathrm{CO}$ для двумерной квадратной решетки в приближении среднего поля задается выражением

$$
T_{\mathrm{CO}}=2\left(\frac{1}{4}-\Delta n^{2}\right) V
$$

При половинном заполнении $(\Delta n=0)$ величина $T_{\mathrm{CO}}=$ $=V / 2$ немного меньше, чем точное значение Онзагера для модели Изинга $(t=0)$

$$
T_{\mathrm{CO}}=\frac{V}{2 \ln (1+\sqrt{2})} \approx 0.567 V .
$$

Несмотря на значительные количественные различия между собой, фазовые диаграммы на рис. 1 обнаруживают явное сходство с фазовой диаграммой для допированных купратов. 

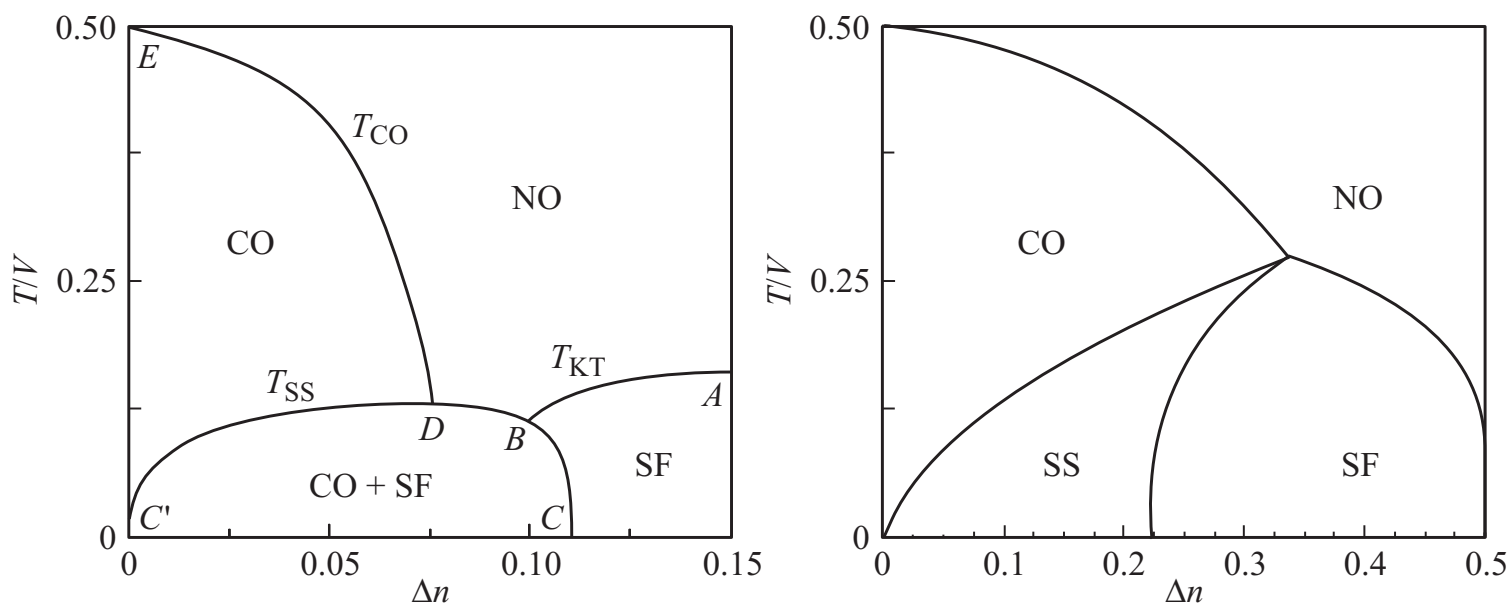

Рис. 1. Фазовая диаграмма системы локальных бозонов на квадратной решетке полученная методом QMC (левая панель) и в приближении среднего поля (правая панель). Линия $A-B$ показывает температуру фазового перехода Березинского-Костерлица-Таулеса; линия $C-B-D-C$ соответствует фазовому переходу первого рода; линия $D-E$ соответствует фазовому переходу второго рода.

Для спиновых систем $s=1 / 2$ СО-фаза соответствует $z$-ориентированной антиферромагнитной (AFM) фазе, с анизотропией типа ,легкая ось“ $\left(J_{n n}^{z}>\left|J_{n n}^{x y}\right|\right)$, SS-фаза соответствует угловой фазе, а SF-фаза - скошенной AFM фазе спинфлоп, имеющей анизотропию типа „Легкая плоскость“" $\left(J_{n n}^{z}<\left|J_{n n}^{x y}\right|\right)$ или находящейся в магнитном поле, ориентированном вдоль оси $z ; \Delta n$ имеет смысл намагниченности. При $J^{z}=\left|J_{n n}^{x y}\right|$ имеет место так называемая точка Гейзенберга, в которой симметрия системы возрастает до группы $O_{3}$, и в этом случае в двумерной системе дальний порядок возникает только при $T=0$.

Спин-флоп переход в квантовых антиферромагнетиках со спином $s=1 / 2$ или эволюция зарядовоупорядоченного основного состояния двумерной системы локальных бозонов при допировании остаются горячо обсуждаемыми фундаментальными проблемами в физике конденсированных сред. Результаты вычислений методом QMC [12] указывают на термодинамическую неустойчивость SS-фазы по отношению к фазовому разделению на CO- и SF-фазы, однако метод QMC не дает наглядной картины разделения фаз. Физика фазового разделения $\mathrm{CO}+\mathrm{SF}$ в модели Бозе-Хаббарда связана с быстрым увеличением энергии однородного СО-состояния при допировании от половинного заполнения из-за больших затрат энергии на „псевдо-спин-флип“. Другими словами, энергетические затраты на допирование бозонами или дырками CO-фазы шахматного типа относительно велики из-за большого вклада бозон-бозонного отталкивания. Следовательно, энергетически выгоднее „выделять“ лишние бозоны (дырки) из СО-состояния и располагать их в конечных кластерах с относительно небольшим числом частиц.

В работе [13] была рассмотрена модель, где бозонное(дырочное) допирование системы локальных бозонов приводит к зарождению топологических дефектов или доменов, в которых сверхтекучая бозе-жидкость и дополнительные бозоны оказываются локализованными в доменных стенках. Такое топологическое разделение фаз $\mathrm{CO}+\mathrm{SF}$ вместо однородной SS-фазы было предложено для описания эволюции локальных бозонов при отклонении $\Delta n$ от половинного заполнения.

Аналогия с магнетиками позволяет однозначно предсказать поведение системы локальных бозонов при допировании. Действительно, допирование бозоном или дыркой „шахматной“ СО-фазы соответствует намагничиванию антиферромагнетика в направлении оси $z$. В однородной $l_{z}$-фазе анизотропного антиферромагнетика типа „легкая ось“ локальные затраты энергии на спин-флип весьма велики. Однако намагничивание анизотропного антиферромагнетика в направлении легкой оси может протекать как фазовый переход первого рода с топологическим разделением фаз из-за существования антифазных доменов. Антифазные доменные стенки являются естественными центрами зародышеобразования для фазы спин-флоп, имеющей бо́льшую поперечную магнитную восприимчивость по сравнению с продольной, что обеспечивает выигрыш в энергии поля. Именно доменные стенки будут определять неоднородную картину намагниченности для такого анизотропного легкоосного антиферромагнетика в относительно слабом внешнем магнитном поле.

В целом, в настоящее время многие вопросы, касающиеся происхождения, детальной структуры фазового состояния $\mathrm{CO}+\mathrm{SF}$ и его эволюции в зависимости от температуры и допирования остаются без ответа.

\section{3. Результаты компьютерного моделирования $2 D$-системы локальных бозонов}

Для минимизации функционала энергии $2 D$-системы локальных бозонов мы использовали высокопроизводительные параллельные вычисления на графических 
картах NVIDIA методом нелинейных сопряженных градиентов [14], а также методом Монте-Карло, чтобы непосредственно наблюдать формирование конфигурации основного состояния с понижением температуры и ее эволюции при отклонении от половинного заполнения, что позволило нам исследовать новые особенности фазовых переходов, в частности, рассмотреть зарождение нерегулярной доменной структуры, локализацию допированных бозонов при отклонении от половинного заполнения и процесс разделения фаз.

Первоначально мы проводили расчет для гамильтониана (2) на квадратной решетке $256 \times 256$ при половинном заполнении $(n=1 / 2)$ при значении межузельного отталкивания $V_{n n}=V=3 t_{n n}$, что является типичным для многих расчетов методом QMC $[10,11,12]$. В дальнейшем мы будем придерживаться обозначений, принятых в этих работах.

Прежде всего, мы рассмотрели формирование фазового состояния в процессе отжига (термализации). Расчет первоначально начинался с высокой температуры, $T \approx 2 T_{c r}$. Отжиг сопровождался образованием неустойчивой доменной структуры СО-фазы с антифазными 180-градусными доменными границами, центр которых характеризуется большим значением параметра SF-порядка, которое уменьшается в глубине области CO-фазы. Это свидетельствует о наличии „хрупкой“ нитевидной (filamentary) сверхтекучести, зародившейся в антифазных границах. Сам термин „нитевидная сверхтекучесть“ является полным аналогом более привычного термина „нитевидная сверхпроводимость“. Мы связываем нитевидную сверхтекучесть с центральной областью антифазной доменной стенки (границы между двумя доменами СО-фазы), которая характеризуется отличным от нуля параметром SF-фазы.

Обычно для малой и средней анизотропии отжиг завершается формированием системы доменов с замкнутыми доменными стенками, которые быстро разрушаются, создавая таким образом однородное однодоменное CO-состояние с едва заметной остаточной неоднородностью. При самых низких температурах при половинном заполнении мы наблюдали формирование почти идеальной зарядово-упорядоченной структуры типа шахматной доски, которая затем не изменялась при увеличении температуры до $T_{\mathrm{CO}}$. Слабое отклонение от половинного заполнения в этом случае приводило к зарождению двух типов топологических дефектов. Первый тип это необычные мелкие наноскопические дефекты с эффективным радиусом в несколько постоянных решетки, содержащие один допированный бозон, которые характеризуются в центре сильным искажением СО-порядка, а на расстоянии трех-четырех координационных сфер от центра - появлением локального сверхтекучего порядка. Второй тип - большие топологические дефекты (капли), имеющие главным образом форму круга и способные вместить множество бозонов. Они состоят из ядра (SF-фазы) и кольцеобразной границы (SS-фазы). Компьютерное моделирование показывает возникновение критического радиуса для устойчивости больших цилиндрических дефектов. С увеличением допирования объемная доля больших дефектов растет, происходит изменение их формы и их слияние вплоть до полного фазового превращения $\mathrm{CO}-\mathrm{SF}$. В то же время стоит отметить сохранение уменьшающейся объемной доли СО-фазы шахматного типа вплоть до больших значений $\Delta n$.

Систематические исследования показали, что в некоторых случаях понижение температуры приводит к формированию устойчивой низкотемпературной доменной структура СО-фазы со страйп-подобными несвязанными (в пределах размера нашей решетки) доменными стенками, ориентированными вдоль главных осей решетки. Наряду с простым однородным („ферромагнитным“) распределением параметра порядка SF-фазы эти одномерные стенки могут иметь необычную многодоменную топологическую структуру с высокой плотностью $2 \pi$-границ, разделяющих одномерные фазовые домены.

Эволюция однородных и страйп-подобных конфигураций основного состояния локальных бозонов для умеренной анизотропии $V=3 t$ при допировании показана на рис. $2, a, b$.

Небольшое отклонение от половинного заполнения при $\Delta n \leq 0.01$ практическине приводит к видимым эффектам, слегка нарушая остаточную неоднородность исходного однородного состояния. Однако при $\Delta n \approx 0.01$ наблюдается внезапное зарождение довольно крупных топологических дефектов (капель), имеющих главным образом круговую форму, и состоящих из SF-ядра и кольцеобразной SS-границы. Эти капли могли вместить все допированные бозоны, сохраняя тем самым однородную окружающую СО-фазу. С увеличением допирования мы приходим к хорошо выраженному разделению фаз c растущей объемной долей больших дефектов, изменением их формы и их слиянием вплоть до полного фазового перехода $\mathrm{CO}-\mathrm{SF}$ вблизи критического значения $\Delta n_{c r} \approx 0.22$. Эволюция страйп-подобной СО-фазы с нитевидной сверхтекучестью при отклонении от половинного заполнения идет по другому сценарию, поскольку допированные бозоны локализуются в центре узких доменных стенок, что приводит к их равномерному уширению вплоть до образования доменов SF-фазы. Интересно, что структура конечной SF-фазы в этом случае зависит от исходной топологической структуры фазового параметра SF-фазы $(\varphi)$ в одномерных доменных границах. На рис. 2, $b$ представлен вариант начального состояния с двумя одномерными доменными стенками с равномерным распределением фазового параметра SF-фазы для нижней стенки и с доменной $2 \pi$-границей, разделяющей одномерные фазовые домены для верхней стенки. Ориентация фазового угла $\varphi$ внутри доменных стенок представлена на рис. 3 для $n=0.02$ и схематично показана на рис. 2, $b$ для $n=0.1$. С отклонением от половинного заполнения в хорошо выраженном режиме разделения фаз мы приходим к системе почти параллельных доменов CO- и SF-фаз, разделенных доменными стенками SS-фазы. Однако регулярная доменная структура становится все более и более неустойчивой 


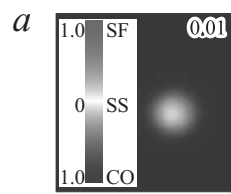

$b$
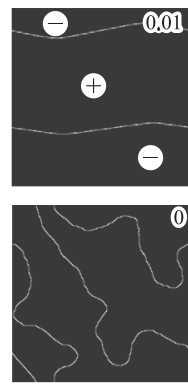
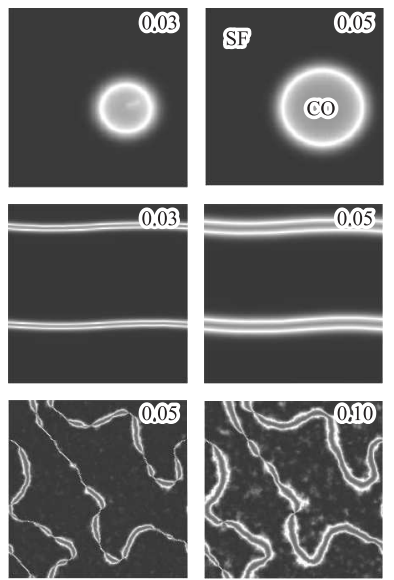
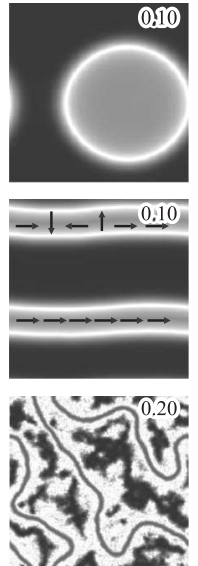
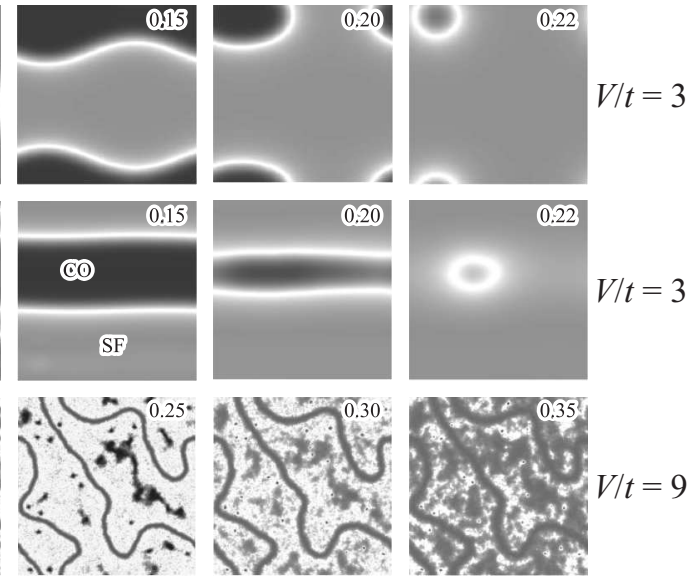

Рис. 2. Эволюция конфигурации основного состояния локальных бозонов при допировании. В правых верхних углах каждой части рис. 2 указано отклонение от половинного заполнения $\Delta n . a)$ Зарождение и рост топологических дефектов (капель), которые могут вместить все допированные бозоны. $b$ ) Уширение доменных границ страйп-подобной фазы СО и возникновение в них нитевидной фазы SF за счет локализации допированных бозонов внутри доменных границ. Знаки „плюс“ и „минус“ указывают на разные CO-домены. При хорошо выраженном разделении фаз возникает системы почти параллельных доменов фаз CO и SF, разделенных доменными границами фазы SS. Ориентация фазового угла $\varphi$ внутри доменных стенок схематически показана для $\Delta n=0.1$. c) При $\Delta n<0.01$ допированные бозоны локализуются в центре узких доменных стенок, разрушая нитевидную сверхтекучесть без видимых преобразований доменов. При дальнейшем допировании доменные границы „разбухают“ неравномерно. Постепенно эти расширения и „капли“ зарождаются и внутри доменов, и в итоге покрывают всю решетку. Топология домена СО-фазы сохраняется до очень выского уровня допирования. Разные оттенки серого соответствуют разным значениям параметров порядка.
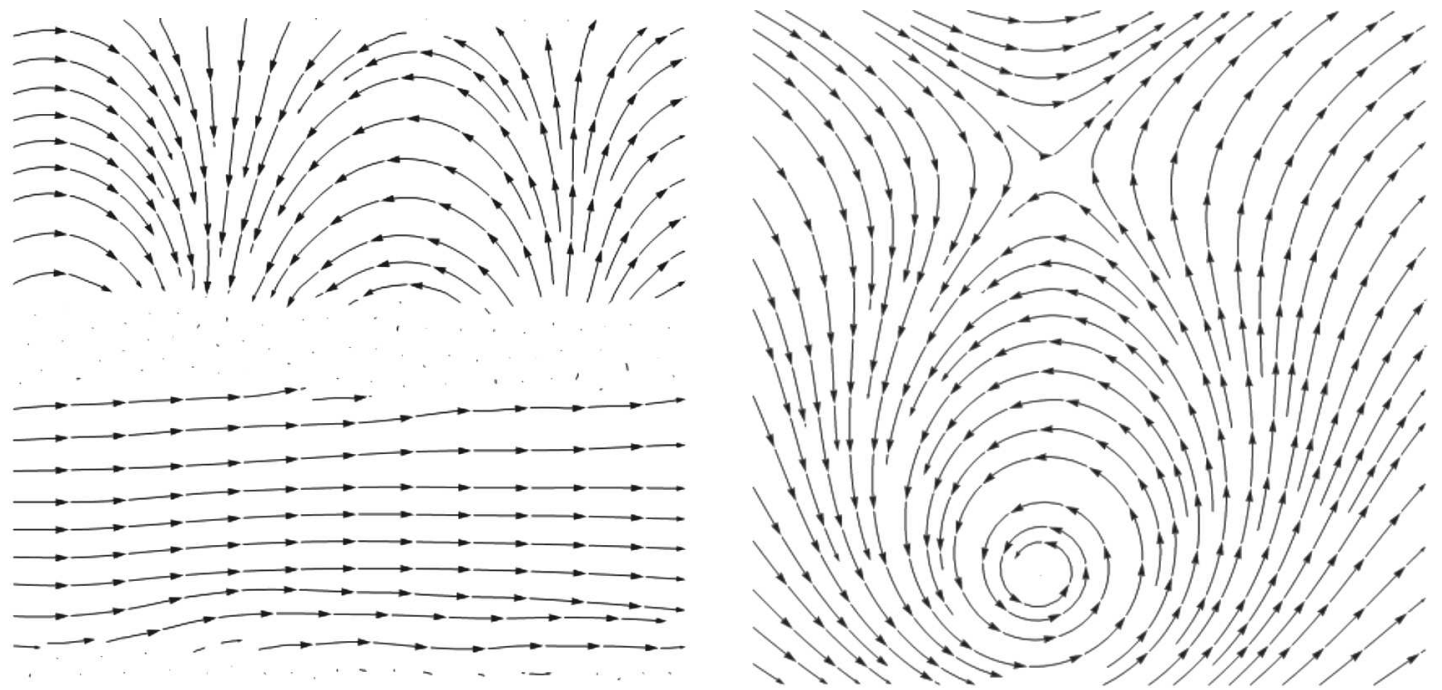

Рис. 3. Левая панель: распределение фазового параметра SF-фазы в одномерных доменах $(\Delta n=0.02)$. Правая панель: распределение параметра порядка SF-фазы в устойчивом топологическом скирмион-подобном дефекте $(\Delta n=0.25)$.

с приближением к точке фазового перехода $\mathrm{CO}-\mathrm{SF}$. Вблизи $\Delta n \approx \Delta n_{c r}$ остатки СО-фазы коллапсируют в скирмион-подобный топологический дефект, который, очевидно, связан с доменной $2 \pi$-границей, разделяющей одномерные фазовые домены (см. рис. 3). Этот точечный дефект выживает вплоть до максимального допирования. Уникальная топология и устойчивость точечного дефекта особенно хорошо демонстрируется распределением параметра SF-фазы на рис. 3. Интересно, что эта эволюция не может быть воспроизведена в обратном порядке, при переходе $\mathrm{SF}-\mathrm{CO}$ с уменьшением допирования. Начальная страйп-структура не восстанавливается, вместо этого мы наблюдали появление необычного скирмионподобного цилиндрического дефекта в матрице CO-фазы, который разрушается с приближением к половинному заполнению.

Компьютерное моделирование фазового перехода $\mathrm{CO}-\mathrm{SF}$ в случае существенно более сильной анизотропии $V=9 t$ обнаружило необычное поведение двумерной системы локальных бозонов. В отличие от предыдущей 
ситуации умеренной анизотропии, процедура термализации приводила в этом случае к появлению хорошо развитой „жесткой“ доменной структуры со 180-градусными доменными стенками, центр которых характеризуется большим параметром SF-фазы. Это свидетельствует о наличии нитевидной сверхтекучести, локализованной в антифазных доменных стенках, существующих внутри СО-фазы. Как и следовало ожидать, ширина стенки увеличивается при увеличении интеграла переноса бозона.

Слабое отклонение от половинного заполнения $(n \leq 0.01)$ не приводит к видимой модификации доменной структуры (см. рис. 2,c), поскольку допированные бозоны локализуются в центре узких доменных стенок, разрушая нитевидную сверхтекучесть без видимых преобразований доменов. Однако дальнейший рост допирования доменных стенок приводит к их сильной трансформации в очень малом диапазоне $\Delta n$. Небольших количеств избыточных бозонов оказывается достаточно для разрушения правильных узких доменных стенок. При этом допирование сопровождалось образованием различных неустойчивых внутриграничных структур, в частности, лестничных структур, образованных бозонными димерами. Затем „полностью заполненная“ доменная стенка регулярно уширяется, так как дополнительные бозоны предпочитают занимать делокализованные состояния вне центра стенки, образуя довольно протяженную оболочку с неоднородным распределением параметров порядка SF- и SS-фаз. При дальнейшем допировании стенки неравномерно „разбухают“, их регулярная форма разрушается. Постепенно эти расширения и „капли“ зарождаются и внутри доменов, и наконец покрывают всю решетку (см. рис. 2,c). Однако топология доменов СО-фазы сохраняется до очень высокого уровня допирования, хотя структура доменных стенок значительно изменяется. Рис. 2, с хорошо иллюстрирует этот своеобразный ,эффект памяти““.

\section{4. Заключение}

Используя высокопроизводительные параллельные вычисления на графических картах NVIDIA методом нелинейных сопряженных градиентов методом классического Монте-Карло мы смогли наблюдать в реальном времени формирование конфигурации основного состояния двумерной системы локальных бозонов с понижением температуры и ее эволюцию с отклонением от половинного заполнения. Компьютерное моделирование позволило нам выявить новые особенности фазового перехода $\mathrm{CO}-\mathrm{SS}-\mathrm{SF}$ для двумерной системы локальных бозонов, которые не могут быть описаны ни в приближении среднего поля, ни методом QMC, в частности, зарождение нерегулярной доменной структуры с нитевидной сверхтекучестью на фоне стабильной СО-фазы. В доменных границах может поддерживаться топологически нетривиальный сверхтекучий фазовый порядок, причем эволюция страйп-структуры может привести к формированию устойчивого топологического дефекта типа скирмиона. Отметим, что в отличие от истинной гейзенберговской спиновой системы, стабильные скирмионы в псевдоспиновой системе $(s=1 / 2)$ могут наблюдаться благодаря ограничению на суммарную $z$-компоненту псевдоспина. Мы считаем, что все обнаруженные особенности универсальны и должны учитываться при обсуждении фазовых переходов и разделения фаз в различных (псевдо)спиновых системах, в частности, в допированных купратах (см., например, [15]). Кроме того, обнаружение стабильных нитевидных сверхтекучих структур в номинально изолирующей СО-матрице открывает новые пути создания конкретных сверхпроводящих гетероструктур, перспективных с точки зрения повышения критической температуры [16].

Один из авторов (АСМ) благодарит А. Bianconi, R. Micnas и A. Менушенкова за полезные дискуссии.

\section{Список литературы}

[1] R. Micnas, J. Ranninger, S. Robaszkiewicz. Rev. Mod. Phys. 62, 113(1990).

[2] A.S. Moskvin. Phys. Rev. B 84, 075116 (2011).

[3] A.S. Moskvin. J. Phys.: Condens. Matter 25, 085601 (2013).

[4] A.S. Moskvin. J. Phys.: Conf. Ser. 592, 012076 (2015).

[5] A.S. Moskvin. ЖЭТФ 148, 549 (2015).

[6] A.S. Moskvin. J. Supercond. Nov. Magn. 29, 1057 (2016).

[7] R. Micnas. Phil. Mag. 95, 622 (2015).

[8] A.P. Menushenkov, A.V. Kuznetsov, K.V. Klementiev, M.Yu. Kagan. J. Supercond. Nov. Magn. 29, 701 (2016).

[9] H. Matsuda, T. Tsuneto. Prog. Theor. Phys. Suppl. 46, 411 (1970).

[10] G.G. Batrouni, R.T. Scalettar. Phys. Rev. Lett. 84, 1599 (2000).

[11] F. Hebert, G.G. Batrouni, R.T. Scalettar, G. Schmid, M. Troyer, A. Dorneich. Phys. Rev. B 65, 014513 (2001).

[12] G. Schmid, S. Todo, M. Troyer, A. Dorneich. Phys. Rev. Lett. 88, 167208 (2002).

[13] A.S. Moskvin. Phys. Rev. B 69, 214505 (2004).

[14] F.N. Rybakov, A.B. Borisov, A.N. Bogdanov. Phys. Rev. B 87, 094424 (2013).

[15] G. Campi, A. Bianconi, N. Poccia, G. Bianconi, L. Barba, G. Arrighetti, D. Innocenti, J. Karpinski, N.D. Zhigadlo, S.M. Kazakov, M. Burghammer, M.V. Zimmermenn, M. Sprung, A. Ricci. Nature 525, 359 (2015).

[16] A. Bianconi. Int. J. Mod. Phys. B 13, 3289 (2000). 